\title{
Sindrome de Down e alterações de vias lacrimais
}

Down syndrome and lacrimal system disorders

\begin{tabular}{llll} 
Suzana & \multicolumn{3}{c}{ Matayoshii } \\
Mariluze & \multicolumn{2}{c}{ Sardinfia } & \\
Alexandra & Lemos & Cozac $^{2}$ \\
Davi Araf & & \\
Eurípedes & da & Mota & Moura
\end{tabular}

Trabalho realizado no Setor de Plástica Ocular, da Clínica Oftalmológica do Hospital das Clínicas da Faculdade de Medicina da Universidade de São Paulo.

${ }^{1}$ Assistente-doutora.

2 Estagiária do $3^{\circ}$ ano.

Médico colaborador pós-graduando (doutorado).

${ }^{4}$ Assistente-doutor e Chefe do Setor de Plástica Ocular. Endereço para correspondência: Rua Dr. César Castiglione Jr. 311 cj.11 - São Paulo (SP)

CEP 02515-000 - São Paulo (SP)

E-mail: smillie@usp.br

Recebido para publicação em 10.06.2002

Aceito para publicação em 30.10.2002

Nota Editorial: Pela análise deste trabalho e por sua anuência na divulgação desta nota, agradecemos aos Drs. Waldir Martins Portellinha e Simone Haberdvellberg von Faber Bison.

RESUMO

Objetivo: Caracterizar as alterações de vias lacrimais em portadores de síndrome de Down. Métodos: Estudo retrospectivo descritivo envolvendo 11 olhos de 8 pacientes com diagnóstico de síndrome de Down, acompanhados no período de 1995 a 2001. Resultados: Todos os pacientes apresentaram quadro de dacriocistite crônica com obstrução no ducto nasolacrimal. Um paciente cursou também com canaliculite por Streptococcus sp e outro paciente apresentava ausência do ponto lacrimal inferior. Cinco pacientes foram submetidos à dacriocistorrinostomia, três à sondagem e intubação das vias lacrimais. A epífora foi resolvida em 4 olhos. Conclusões: Existem poucos relatos acerca do comprometimento das vias lacrimais em pacientes com síndrome de Down. A caracterização e a resolução cirúrgica do mecanismo obstrutivo lacrimal são importantes, uma vez que há interferência na qualidade de vida desses pacientes. No presente estudo, verificou-se que a obstrução da via lacrimal ocorreu no ducto nasolacrimal. O prognóstico cirúrgico foi ruim em $83,4 \%$ das intubações e em 57,2\% das dacriocistorrinostomias. Causas do insucesso cirúrgico podem estar relacionadas à maior fibrose pós-operatória nesses pacientes e à dificuldade de colaboração dos mesmos no controle pós-cirúrgico. Estudos posteriores são necessários para verificação das presentes observações.

Descritores: Síndrome de Down; Doenças do aparelho lacrimal; Dacriocistite; Dacriocistorrinostomia; Intubação/métodos

INTRODUÇÃO

A síndrome de Down constitui a anormalidade cromossômica mais comum do homem ${ }^{(1)}$. No Brasil sua incidência é cerca de 1,13:1.000 nascidos vivos $^{(2)}$. Em 1959, com o advento da cariotipagem, foi descrita a principal causa da síndrome, a trissomia do cromossomo $21^{(3)}$.

O número de portadores desta síndrome vem aumentando possivelmente pelo aumento de gestações em mulheres acima de $35 \operatorname{anos}^{(4)}$ e pelo aumento da longevidade destes pacientes ${ }^{(5)}$.

São muitas as alterações oftalmológicas encontradas nestes pacientes, entretanto pouco se comenta em relação ao acometimento das vias lacrimais.

O objetivo do presente estudo é avaliar as características da obstrução das vias lacrimais nos portadores da síndrome de Down, bem como discutir sua abordagem terapêutica e os resultados cirúrgicos.

\section{MÉTODOS}

Trata-se de um estudo retrospectivo envolvendo oito pacientes acompanhados no período de 1995 a 2001. 
Todos os pacientes tinham diagnóstico clínico de síndrome de Down e queixa de epífora e/ou secreção ocular crônica.

Foram revistos os prontuários e compilados dados relacionados às vias lacrimais. Todos os pacientes apresentavam exame oftalmológico incluindo biomicroscopia, refração estática, fundoscopia, teste de Zappia Milder e dacriocistografia.

Os pacientes foram submetidos a dois tipos de procedimentos: intubação de via lacrimal com silicone e dacriocistorrinostomia com ou sem a utilização de silicone como modelador no pós-operatório.

A intubação lacrimal foi realizada utilizando-se o método de Crawford ${ }^{(6)}$. As extremidades do silicone foram anodadas com fio mononylon 5-0 e fixadas na porção lateral interna do vestíbulo nasal.

A técnica de dacriocistorrinostomia empregada neste estudo foi a externa ${ }^{(7)}$, com confecção de retalhos do saco lacrimal e mucosa nasal em U (2 suturas dos retalhos com vicryl 6-0).

Em relação aos critérios de indicação cirúrgica, as crianças abaixo de 4 anos de idade e com saco lacrimal pequeno foram submetidas à sondagem lacrimal e intubação com silicone. Acima desta idade ou nos casos de saco lacrimal dilatado, os pacientes foram submetidos à dacriocistorrinostomia. A utilização de silicone nas dacriocistorrinostomias era feita nos casos de dificuldade de obtenção de retalhos adequados e naqueles casos de reoperações ${ }^{(7)}$.

O critério de cura foi clínico (ausência de sinais clínicos de secreção ou epífora, confirmados pelo teste de Zappia Milder). A patência da via, através da irrigação lacrimal com solução fisiológica a $0,9 \%$, foi realizada nos casos onde havia colaboração do paciente. A comprovação de recidiva foi feita através de dacriocistografia.

\section{RESULTADOS}

A tabela 1 resume os principais achados dos pacientes do estudo.

Oito pacientes portadores de síndrome de Down foram estudados, sendo que 6 deles eram crianças abaixo de 8 anos. A média do seguimento ambulatorial foi de 3 anos. Os pacientes foram referidos para tratamento devido à queixa de epífora e/ou secreção, com predomínio de epífora sobre o quadro de secreção. Cinco dos casos eram unilaterais e três bilaterais. Três pacientes $\left(\mathrm{n}^{0} 1,3\right.$ e 5$)$ apresentavam também blefarite severa bilateral. A blefarite da paciente de $\mathrm{n}^{0} 2$ melhorou drasticamente após a resolução da obstrução lacrimal.

Em relação à localização do processo obstrutivo, as dacriocistografias e as observações no per-operatório mostraram que todos os pacientes apresentavam obstrução entre o saco lacrimal e a porção mais alta do ducto nasolacrimal, sendo que o paciente de $\mathrm{n}^{0} 8$ apresentava ainda ausência do ponto lacrimal inferior. As dacriocistografias mostraram saco lacrimal de tamanho normal ou com pouca dilatação.

O caso de $n^{0} 5$, além da dacriocistite apresentava canaliculite inferior direita (Streptococcus sp), constatada no exame per-operatório, procedeu-se à canaliculotomia além da dacriocistorrinostomia. Este paciente havia sido submetido à facectomia de olho direito cerca de 2 meses antes de ser referido para tratamento das vias lacrimais.

A paciente de $n^{0} 3$ foi submetida à intubação de vias lacrimais bilateralmente, com melhora da epífora e secreção durante cerca de 30 dias, após o qual teve recidiva bilateral, sendo então indicada dacriocistorrinostomia. $\mathrm{O}$ caso de $\mathrm{n}^{0} 8$ foi submetido à intubação monocanalicular, devido à ausência do ponto lacrimal inferior.

Foram realizadas 6 intubações, das quais apenas 1 foi bem sucedida; 7 dacriocistorrinostomias primárias das quais 3 tiveram bom resultado. As reoperações foram realizadas em 2 pacientes, com sucesso em apenas em 1 deles.

\section{DISCUSSÃO}

O avanço tecnológico da medicina tem propiciado condições para melhor avaliação e tratamento das alterações sistêmicas na síndrome de Down, tais como cardiopatias congênitas, pneumonias e leucemias agudas, principais causas de morte nesses pacientes ${ }^{(5)}$. $\mathrm{O}$ aumento da longevidade se associa com uma maior atenção às outras manifestações da síndrome, na perspectiva de melhora da qualidade de vida desses pacientes.

As alterações oculares são encontradas em quase todos os pacientes com síndrome de Down, e, embora não ofereçam risco de vida, podem comprometer consideravelmente a qualidade de vida deles, sendo as complicações da cirurgia de catarata e o ceratocone agudo, as principais causas de cegueira ${ }^{(8)}$.

Existem poucos trabalhos a respeito do comprometimento da via lacrimal em pacientes com síndrome de Down. Catalano, em 1990, refere em seu trabalho que a obstrução do ducto nasolacrimal é um achado raro e provavelmente não relacionado à síndrome $\mathrm{e}^{(9)}$, enquanto Cunha et al, em 1986, relata uma prevalência de $30 \%{ }^{(2)}$. Mais recentemente, em 2000, Lueder refere obstrução do ducto nasolacrimal como uma ocorrência freqüente nos pacientes com trissomia $21^{(10)}$.

A síndrome de Down tem sido citada, mas não caracterizada, como uma das doenças na qual pode ser encontrada obstrução congênita do recém-nascido ${ }^{(11)}$. Nesta casuística, embora a maior parte dos pacientes fossem crianças $(75 \%)$, os achados dacriocistográficos e cirúrgicos demonstraram que a obstrução lacrimal ocorreu entre o saco lacrimal e o ducto nasolacrimal, caracterizando obstrução que se observa mais em adultos.

A blefarite é uma alteração bastante descrita entre os pacientes da síndrome de Down $(30 \%)^{(2)}$, podendo estar associada à obstrução das vias lacrimais, como aconteceu nos casos 4, 5 e 6 . Fato importante a ser ressaltado é que tanto a blefarite, quanto a obstrução das vias lacrimais, podem se apresentar com sintomas semelhantes, dificultando o diagnóstico correto desta última. A impressão é que a prevalência de obstrução lacrimal nesses pacientes talvez seja subestimada na literatura.

O paciente de $\mathrm{n}^{0} 7$ foi referido para tratamento da dacriocistite após facectomia. É oportuno considerar a necessidade de diagnóstico e tratamento de afecções infecciosas de vias lacrimais previamente a cirurgias intra-oculares a fim de se evitar endoftalmite pós-operatória ${ }^{(12)}$. 


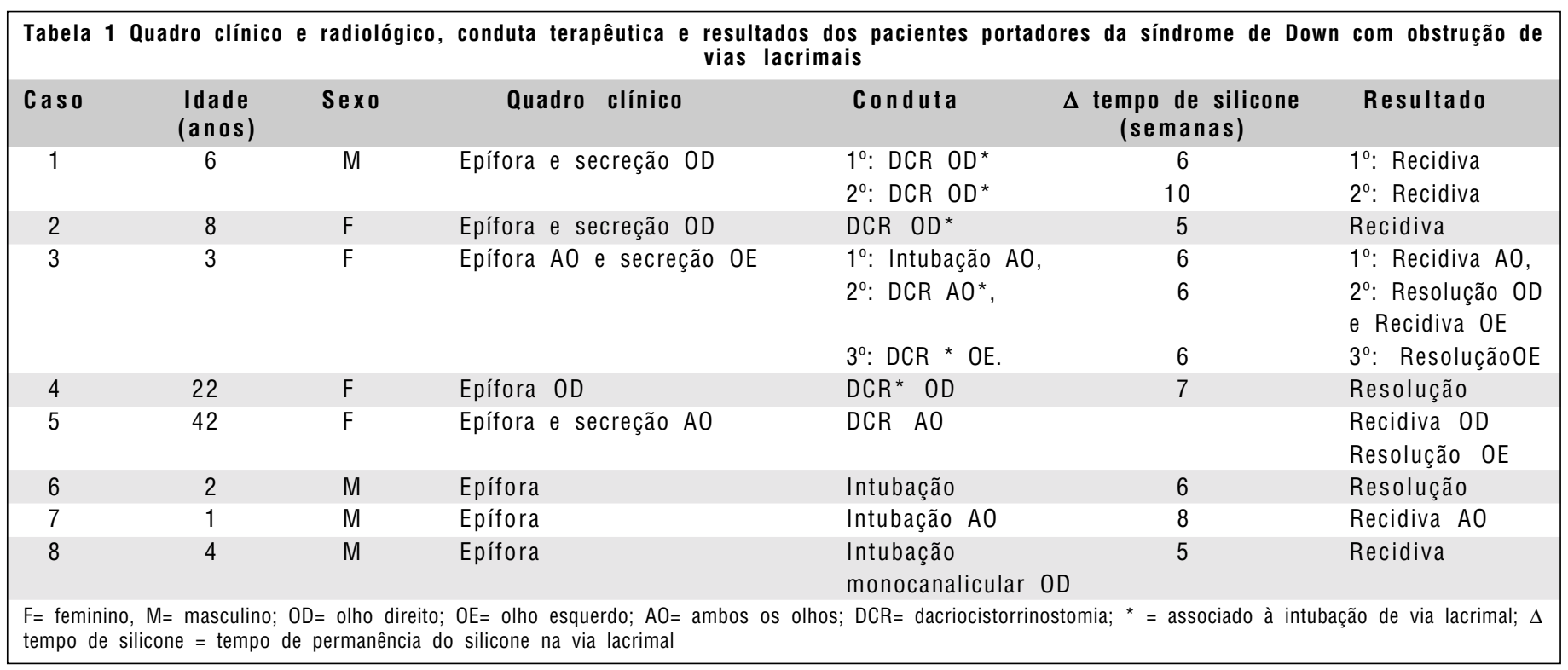

Em relação ao tratamento da obstrução lacrimal, o enfoque é basicamente cirúrgico. No caso da obstrução congênita, preconiza-se a sondagem simples ou a sondagem associada à intubação com silicone. Já a obstrução do terço superior do ducto nasolacrimal é tratada com dacriocistorrinostomia com ou sem intubação ${ }^{(13)}$. Um outro método, descrito por Lueder, foi a utilização de cateter com balão de dilatação com bons resultados ${ }^{(10)}$.

Os casos 3, 6, e 8 foram submetidos à sondagem e intubação. A obstrução lacrimal congênita tem um índice alto de sucesso terapêutico ${ }^{(11)}$. O fato de apresentarem obstrução no terço superior do ducto nasolacrimal provavelmente teve influência na recorrência dos sintomas após o tratamento(13).

O índice de sucesso cirúrgico final foi de $16,6 \%$ nos casos submetidos à intubação e de $42,8 \%$ nas dacriocistorrinostomias primárias. A eficácia reduzida da intubação lacrimal nos casos deste estudo desencoraja este procedimento em pacientes com síndrome de Down.

Em relação à dacriocistorrinostomia convencional, há significativa diferença com o resultados numa população geral, onde o índice de sucesso é em torno de 90 a $100 \%{ }^{(14)}$; entretanto atualmente parece ser a melhor possibilidade terapêutica.

Sabe-se ainda que a dacriocistorrinostomia em população pediátrica tem uma maior taxa de insucesso que no adulto ${ }^{(15)}$. Fatores anatômicos (posicionamento anterior das células etmoidais, a presença do centro de crescimento dos ossos faciais), dificuldade técnica (posicionamento inapropriado da osteotomia) e fatores relacionados com o processo de cicatrização (tendência à maior fibrose e formação de tecido de granulação no sítio da osteotomia) podem explicar tais resultados ${ }^{(15)}$.

O baixo índice de sucesso cirúrgico observado no presente estudo poderia também se relacionar com o tipo de paciente em questão, envolvendo as alterações sistêmicas presentes nestes pacientes e a maior tendência a infecções por alterações em seu sistema imunológico principalmente por defeitos de maturação das células $\mathrm{T}^{(16-17)}$. A pouca colaboração na realização de cuidados locais pós-operatórios (irrigação de vias lacrimais, exame endoscópicos e limpeza de cavidade), também podem interferir no prognóstico cirúrgico, pois tais procedimentos são considerados atualmente importantes para o bom funcionamento da fístula cirúrgica ${ }^{(18)}$.

\section{CONCLUSÕES}

Existem poucos relatos acerca do comprometimento das vias lacrimais em pacientes com síndrome de Down. A caracterização e a resolução cirúrgica do mecanismo obstrutivo lacrimal são importantes, uma vez que há interferência na qualidade de vida desses pacientes. No presente estudo, verificouse que a obstrução da via lacrimal ocorreu no ducto nasolacrimal. O prognóstico cirúrgico foi ruim em $83,4 \%$ das intubações e em 57,2\% das dacriocistorrinostomias. Causas do insucesso cirúrgico podem estar relacionadas à maior fibrose pós-operatória nesses pacientes e à dificuldade de colaboração dos mesmos no controle pós-cirúrgico. Estudos posteriores são necessários para verificação das presentes observações.

\section{ABS T RAC T}

Purpose: To identify the alterations of the lacrimal system in patients with Down syndrome. Methods: A descriptive and retrospective survey covering eleven eyes in eight patients, diagnosed as Down syndrome, attended in the period from 1995 to 2001. Results: All patients had chronic dacryocystitis with obstruction of the nasolacrimal duct. One patient also presented canaliculitis by Streptococcus sp and congenital absence of the lower lacrimal punctum was observed in another patient. Five patients were submitted to dacryocystorhinostomy, probing and intubation was performed in three 
patients. The complaints of epiphora were solved in four eyes. Conclusions: There are few accounts about lacrimal system disorders in patients with Down syndrome. The identification and surgical resolution of lacrimal obstruction is important, since it interferes with the quality of life of these patients. In the present study, it was verified that the lacrimal obstruction occurred in the nasolacrimal duct, like that which occurs in adults. The surgical prognosis was bad in $83.4 \%$ of intubations and in $57.2 \%$ of dacryocystorhinostomies. The reasons for the unsuccessful surgery may be related to a greater postoperative fibrosis in these patients, and the difficulty of obtaining their collaboration after surgery. Further studies are necessary to support the present observation.

Keywords: Down syndrome; Lacrimal apparatus diseases; Dacryocystitis; Dacryocystorhinostomy; Intubation

\section{REFERENCIAS}

1. Frota-Pessoa O, Otto PA, Otto PG. Mongolismo. In: Frota-Pessoa O, Otto PA, Otto PG, editores. Genética clínica. São Paulo: Francisco Alves; 1987. p.143-57

2. Cunha, RNP, Moreira JBC. Manifestações oculares em crianças e adolescentes com Síndrome de Down. Arq Bras Oftalmol 1995;58:152-7.
3. Lejune J, Gautier M, Turpin R. Les chromossomes humains en culture de tissues. Comples Rendus Seances Acad 1959;248:602-3.

4. Huether CA. Projection Down's syndrome births in the United States 1979-2000, and the potential effects of prenatal diagnosis. Am J Public Health 1983;73:1186-9.

5. Bairde PA, Sadovnick AD. Life expectancy in Down syndrome. J Pediatr 1987;110:849-54.

6. Kraft SP, Crawford JS. Silicone tube in disorders of the lacrimal system in children. Am J Ophtalmol 1982;94:290-9.

7. Moura EM, Couto ASJ, Melo AF, Silva, JÁ Dacriocistorrinostomia. In: Soares EJC, Gonçalves JOR. Cirurgia plástica ocular. São Paulo: Roca; 1997. p.494-506

8. Cullen JF. Blindness in mongolism (Down's syndrome). Br J Ophthalmol 1963;47:331-3.

9. Catalano RA. Down syndrome. Surv Ophthalmol 1990;34:385-98.

10. Lueder GT. Treatment of nasolacrimal duct obstruction in children with trissomy 21. J AAPOS 2000;4:230-2.

11. França VP. Obstrução lacrimal do recém-nascido. In: Soares EJC, Moura EM, Gonçalves JOR. Cirurgia plástica ocular. São Paulo: Roca; 1997. p.479-92.

12. Wesley RE. Lacrimal disease. Curr Opin Ophthalmol 1994;5:78-83.

13. Soares EJC, França VP. Importância da dacriocistografia no tratamento da obstrução lacrimal do recém-nascido. Arq Bras Oftalmol 1987;50:225-36.

14. Woog JJ, Kennedy RH, Custer PL, Kartreider SA, Meyer DR, Camara JG. Endonasal dacryocystorhinostomy.- A report by the American Academy of Ophthalmology. Ophthalmology 2001;108:2369-77.

15. Nowinski TS, Flanagan, JC, Mauriello J. Pediatric dacryocystorhinostomy. Arch Ophthalmol 1985;103:1226-8.

16. Levin S, Nir E, Mogilner BM. T system immune deficiency in Down's syndrome. Pediatrics 1975;56:123-6.

17. Ugazio AG.Down's syndrome: problems of immunodeficiency. Hum Genet 1981; Suppl 2:33-9

18. Linberg JV. Lacrimal surgery. In: Linberg JV. Endoscopy. Nova York: Churchill Livingstone; 1998;274-314.

\title{
XXXIX Reunião Anual do Centro de Estudos Oftalmológicos "Cyro de Rezende"
}

\author{
5 e 6 de dezembro de 2.003 \\ Departamento de Oftalmologia e Otorrinolaringologia e \\ Cirurgia de Cabeça e Pescoço da Faculdade de \\ Medicina de Ribeirão Preto
}

RIBEIRÃO PRETO - SP

INFORMAÇÕES: E-mail: jmcatique@yahoo.com.br 